\title{
LINBURG-COMSTOCK SYNDROME: A CASE REPORT
}

Sánchez-Ponce $\mathrm{G}^{1}$, Mas Martínez Elia ${ }^{1}$, Sánchez-Jiménez $\mathrm{A}^{2}$, Zaballos Carpintero $\mathrm{V}^{1}$.

\section{$\underline{\text { Abstract }}$}

La variante de Linburg-Comstock consiste en una conexión tendinosa anómala entre los tendones flexor pollicislongus (FPL) y más frecuentemente el flexor digitorum profundus (FDP), provocando incapacidad para la flexión aislada del primer dedo. Se puede manifestar como dolor intermitente en la superficie volar del antebrazo, entumecimiento en los dedos, hormigueo y dolor nocturno. Puede tratarse con éxito mediante escisión quirúrgica y para su diagnóstico cabe destacar la utilidad de la ecografía dinámica musculoesquelética, disponible en nuestras consultas, como instrumento útil y eficiente para el diagnóstico de sospecha de la misma

Keywords: Linburg-Comstock, síndrome, anomaly, dynamic ultrasound

${ }^{1}$ Corresponding Author: Sánchez-Ponce G; Arnau Vilanova Hospital.

sanchez.genop3@gmail.com

Carrer de SantClement, 12, 46015 València, Valencia

${ }^{2}$ La Fe Hospital, Avd Fernando Abril Martorell 106, 46026 Valencia 


\section{INTRODUCCIÓN}

Varios anatomistas describieron la conexión tendinosa anómala entre el flexor policislongus (FPL) y el flexor digitorumprofundus (FDP) a final del siglo XIX [1,2].

En 1979, Linburg and Comstock publicaron por primera vez su frecuencia en humanos. Según estos autores, esta anomalía se presenta en un $31 \%$ de la población de forma unilateral y en un $25 \%$ en cadáveres. La incidencia varía según los estudios de un 12,5 al 36\%. Esta interconexión tendinosa es la variación de Linburg-Comstock (LCV) que es asintomática y cuando se manifiesta se denomina síndrome de LinburgComstock (LCS) [3].

Es interesante remarcar que, aunque esta variación en bastante prevalente hay pocos estudios clínicos que la estudien. En un metaanálisis reciente, se encontró mayor prevalencia en población turca que en europeos (22.2 vs $15.2 \%$ ). En la población española se presenta en un $34.5 \%$ mientras que en africanos solo en un $8.8 \%$ [4].

La prevalencia del lado derecho fue mayor en hombres, mientras que en el lado izquierdo fue más frecuente en mujeres, pero la diferencia entre ellas no fue estadísticamente significativa. Aunque todos los dedos podrían estar involucrados, se presentaba con más frecuencia en el índice[5].

Linburg y Comstock describieron un test para el diagnóstico de esta anomalía, en el que el examinador pasivamente bloquea la flexión de los dedos, mientras el paciente flexiona el pulgar hacia la base del $5^{\circ}$ dedo. Un test positivo se caracteriza por la restricción activa de la flexión del pulgar con dolor asociado o calambres en la zona palmar y radial de la muñeca, o distal del antebrazo [3].

Un test alternativo, es que el paciente al flexionar el pulgar flexiona el índice. Si con la extensión pasiva del índice aparece dolor se considera el test positivo [6].

El tratamiento conservador como: férulas, AINES, ejercicios de estiramiento y las inyecciones de esteroides locales, no han mostrado eficacia [7].

La cirugía mediante la liberación de las conexiones tendinosas en el LCS se ha demostrado eficaz[3].

\section{CASO CLÍNICO}

Presentamos el caso de una mujer de 42 años que acude a consultas externas de Rehabilitación, remitida desde su Médico de Atención Primaria por cervicobraquialgia derecha de 6 meses de evolución, sin otros antecedentes patológicos de interés. Diestra y de profesión policía (uso de armas).

La paciente refiere dolor cervical irradiado a miembro superior derecho (MSD) sin distribución radicular específica, que mejora tras tratamiento fisioterápico.

Persisten molestias inespecíficas a nivel de antebrazo y muñeca derechas con dolor a la presión sobre zona musculatura flexora a nivel tercio medio de antebrazo, junto con la aparición de la flexión involuntaria del segundo dedo al flexionar de forma activa la falange distal del pulgar de ambas manos con predominio derecho (Fig. 1 y 2).

Sin encontrar otros hallazgos en la exploración. 

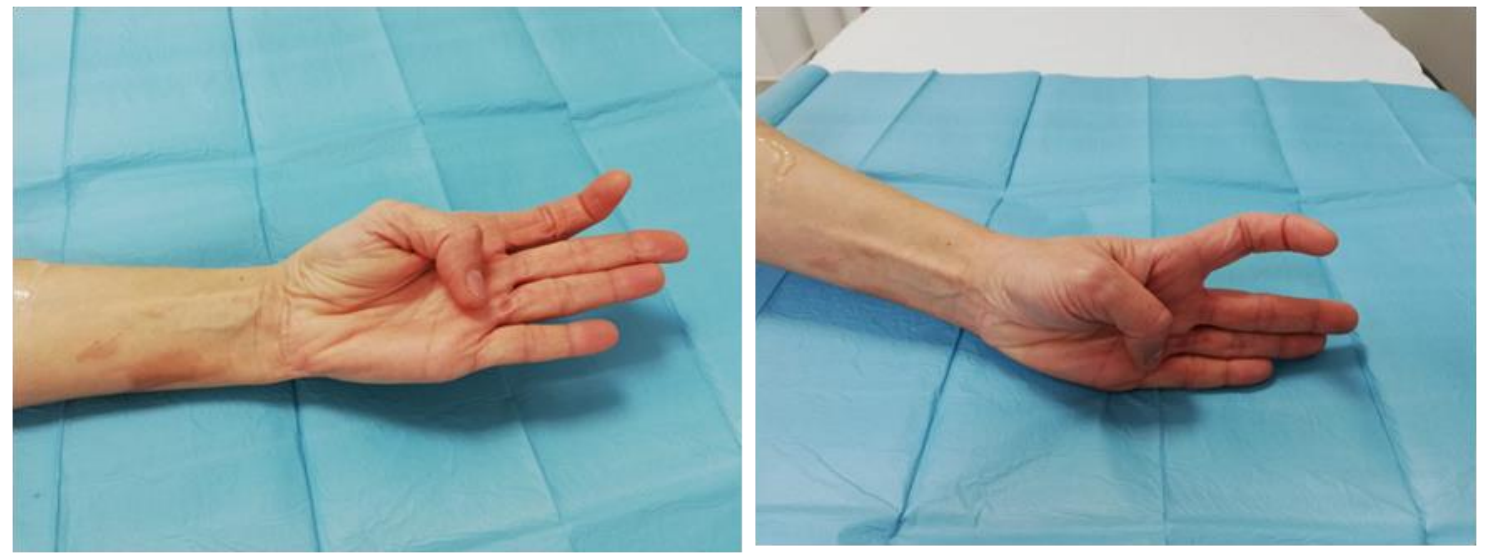

Figura 1 y 2: En las imágenes durante la exploración: aparición de flexión conjunta del segundo dedo al realizar la flexión activa voluntaria de la falange distal del pulgar

Como único antecedente en semanas previas destacar la realización de una electromiografía de miembros superiores sin alteraciones de significación.

La paciente aporta resonancia magnética (RM) de mano (realizada en Mutua laboral) sin hallazgos patológicos.

Dada la sintomatología, se realiza en consulta Ecografía con transductor lineal (Frecuencia $12 \mathrm{mHz}$ ) de MSD, dónde la exploración dinámica a nivel de tercio mediodistal de cara volar de antebrazo es sugestiva de unión miotendinosa del FPL y del FDP, al solicitar flexión activa de falange distal del pulgar se observa el arrastre del FDP (figura 3 y vídeo), por lo que se decide ampliar el estudio con RM de antebrazo en la que únicamente se aprecian cambios de sinovitis en cápsula dorsal del carpo sin otros hallazgos.

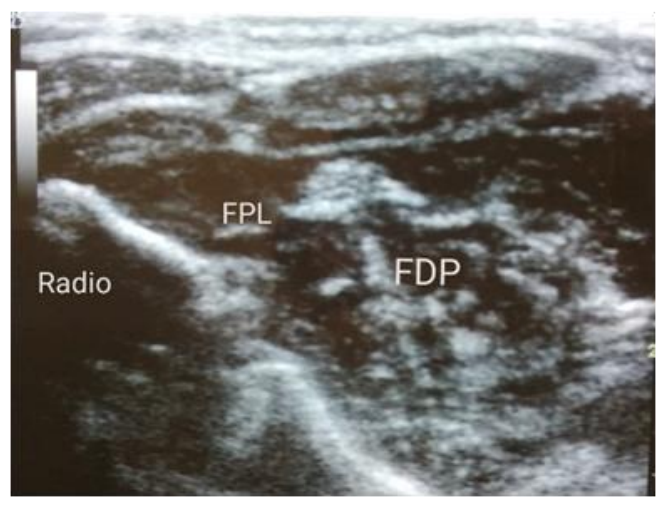

Figura 3: Imagen ecográfica de cara volar de tercio medio-distal de antebrazo en corte transversal. Video: incapacidad del movimiento independiente de los tendones FPL y el FDP

En el servicio de Radiología, realizan ecografía dinámica de antebrazo derecho encontrando la sospecha de la unión de los tendones FPL y flexor del índice sin evidenciar vínculo entre ambos flexores ni tampoco cambios inflamatorios. Se realiza infiltración bajo control ecográfico con lidocaína y triamcinolona en compartimentos flexores de primer y segundo dedo de mano derecha sin mejoría clínica. 
Ante los hallazgos encontrados con la ecografía dinámica se remite al servicio de COT para valoración quirúrgica, indicando tenolisis quirúrgica. En la cirugía se identificaron el FLP y el FP del segundo dedo, que presentaban una víncula de tejido fibroso entre ambos a nivel del túnel del carpo y en el inicio del mismo.

Se resecó la víncula, comprobando independencia para la flexión de ambos tendones por separado.

La paciente posteriormente inicia tratamiento rehabilitador con movilizaciones activo-asistidas, despegamiento de cicatriz adherida y fortalecimiento de musculatura de antebrazo y mano.

A los 7 meses tras la cirugía la paciente se encuentra asintomática, incluso ha reanudado su actividad laboral sin limitaciones.

\section{DISCUSIÓN}

En la población general podemos encontrar diferentes variantes en la musculatura a nivel de la muñeca en muchas ocasiones asintomáticas, pero en otras pueden ser causa de discapacidad.

Cuando está presente la LCV puede dar sintomatología antes de manifestarse el LCS. Puede comenzar con dolor en antebrazo, edema, tenosinovitis de flexores y síntomas de túnel carpiano secundario [6].

Uno de los factores que se barajan como causa de este síndrome es la movilidad repetitiva de los dedos, Lombardi et al lo justifican como una tenosinovitis debida a la tracción repetitiva e irritación [6].

Según Rennie and Muller no encontraron esta relación entre la actividad repetitiva y la tenosinovitis causante de la adherencia tendinosa [8]. En nuestro caso podríamos pensar en una tenosinovitis por sobre uso laboral, pero la sintomatología se presentó de forma bilateral.

La ecografía es una técnica de importante valor en el diagnóstico de enfermedades musculo-esqueléticas. Es disponible, barata, no invasiva, permite una valoración rápida y dinámica de las estructuras.

De hecho, algunos autores señalan que la ecografía dinámica de alta resolución debería usarse en el diagnóstico del LCS, tiene algunas ventajas sobre la RM para identificar interconexiones tendinosas o tenosinoviales; como la de ser más sensible para reconocer interconexiones menores de 1 $\mathrm{mm}$ de grosor, permite una valoración dinámica, no hay artefactos con el material metálico y además se puede comparar con el antebrazo contralateral [9].

Sabiendo que la LCV podría observarse a nivel del antebrazo, muñeca y mano $[3,6]$, una localización preoperatoria con RM sería útil antes del tratamiento quirúrgico [9].

En el caso presentado todas las pruebas diagnósticas realizadas fueron negativas, fue la ecografía dinámica la que nos hizo sospechar de la existencia del LCS al observar la incapacidad del movimiento independiente del FLP y FDP.

Según diferentes autores, el tratamiento consiste en la escisión quirúrgica con muy buenos resultados $[3,6]$. En nuestra paciente el tratamiento conservador fracasó y fue el tratamiento quirúrgico junto con la rehabilitación posterior lo que consiguió la mejora del dolor y la vuelta a su actividad laboral incluso al uso de armas. 


\section{REFERENCIAS BIBLIOGRÁFICAS}

1. Macalister A. Additional observations on muscular anomales in human anatomy. (Third Series) With a catalogue of the principal muscular variations. Trans Roy IrishAcad. $1875 ; 25: 1-134$

2. Testut L. Les anomalies musculaireschez I'homme expliquées par l'anatomie comparée. Leur importance en anthropologie. G. Masson, Paris.1884; 4:454-509Disponible en :https://archive.org/stream/lesanomalie smusc00testuoft\#page/n5/mode/2up

3. Linburg RM, Comstock BE. Anomalustendon slips from the flexor pollicis longus to the flexor digitorum profundus. J Hand Surg Am. 1979; 4:79-83.

4. Yammine $K$, Eric $M$, Linburg-Comstock variation and síndrome. A meta-analysis. SurgRadiolAnat 2017 40(3):289-296. Disponible en: https://doi.org/10.1007/s00276-017-19571.

5. Eric M, Yammine K, Gosh V, Feigl G, Maric $D$, Prevalence of the Linburg-Comstock variation through clinical evaluation. SurgRadiolAnat 2019 Nov;41(11):1307$1314 . \quad$ Disponible en: https://doi.org/10.1007/s00276-01902265-0.

6. Lombardi RM, Wood MB, Linscheid RL. Symptomatic restrictive thumb-index flexor tenosynovitis: incidence of musculotendinousanomalies and results of treatment. J Hand Surg Am. 1988; 13:325-328.
7. Yoon Hong-Kee, Kim ChangHyun.Linburg-Comstock syndrome involving four fingers: A case report and review of the literatura. JPRAS [Internet] 2013 66, 1291-1294. Disponible en: https://dx.doi.org//10.1016/j.bjps.2012.12.0 32

8. Rennie WR, Muller H. Linburg síndrome. J Hand Surg Am. 1998; 41(4):306-308.

9. Smith J, Finnoff JT. Diagnostic and interventional musculoskeletal ultrasound: part 1. Fundamentals PM\&R. 2009;1(1):64-75. 